\title{
Salvage Versus Primary Robot-assisted Radical Prostatectomy: A Propensity-matched Comparative Effectiveness Study from a High-volume Tertiary Centre
}

\author{
Arjun Nathan ${ }^{a, b, \dagger, *}$, Monty Fricker $^{c, \dagger}$, Ruben De Groote ${ }^{d}$, Amandeep Arora $^{e}$, Yuzhi Phuah $^{b}$, \\ Kiran Flora ${ }^{b}$, Sonam Patel ${ }^{b}$, Veeru Kasivisvanathan ${ }^{a, b}$, Ashwin Sridhar $^{a}$, Greg Shaw $^{a}$, \\ John Kelly ${ }^{a}$, Tim Briggs ${ }^{a}$, Prabhakar Rajan ${ }^{a, f}$, Prasanna Sooriakumaran ${ }^{a, g}$, Senthil Nathan ${ }^{a}$ \\ ${ }^{a}$ Department of Uro-oncology, University College London Hospitals NHS Foundation Trust, London, UK; ${ }^{\mathrm{b}}$ University College London, London, UK; ${ }^{\mathrm{c}}$ University \\ of Newcastle, Newcastle, UK; ${ }^{\mathrm{d}}$ Department of Urology, Onze Lieve Vrouw Hospital Aalst, Aalst, Belgium; ${ }^{\mathrm{e}}$ Department of Urology, Tata Memorial Hospital, \\ Mumbai, India; ${ }^{\mathrm{f}}$ Barts Cancer Institute, CR-UK Barts Centre, Queen Mary University of London, London, UK; ${ }^{\mathrm{g}}$ Nuffield Department of Surgical Sciences, \\ University of Oxford, Oxford, UK
}

Article info

Article history:

Accepted March 6, 2021

Associate Editor:

Guillaume Ploussard

Keywords:

Erectile dysfunction

Radiotherapy

Recurrence

Prostatectomy

Urinary incontinence

\begin{abstract}
Background: Salvage robot-assisted radical prostatectomy (sRARP) is a potential treatment option for locally recurrent prostate cancer (PCa) after nonsurgical primary treatment. There are minimal data comparing outcomes between propensity-matched sRARP and primary robot-assisted radical prostatectomy (RARP). Objective: The primary objective is to compare perioperative, oncological, and functional outcomes of sRARP with primary RARP, and the secondary is to compare outcomes between sRARP after whole and focal gland therapy.

Design, setting, and participants: A 1:1 propensity-matched comparison was carried out of 135 sRARP cases with primary RARP cases from a cohort of 3852 consecutive patients from a high-volume tertiary centre.

Outcome measurements and statistical analysis: Perioperative, oncological, and functional outcomes including complication rates, positive surgical margins, biochemical recurrence (BCR), continence, and erectile dysfunction (ED) were retrospectively collected.

Results and limitations: There were no significant differences in patient characteristics between SRARP and primary RARP groups. In the salvage and primary groups, median (interquartile range) follow-up periods were 521 (304-951) and 638 (394-951) d, grade III-V Clavien-Dindo complication rates were $1.5 \%$ and $0 \%(p$ $=0.310)$, BCR rates were $31.9 \%$ and $14.1 \%(p<0.001)$ at the last follow-up, pad-free continence rates were $78.8 \%$ and $84.3 \%$ at $2 \mathrm{yr}(p=0.337)$, and ED rates were $94.8 \%$ and $76.3 \%(p<0.001)$, respectively. Comparing the whole and focal gland groups, BCR rates

Both authors contributed equally to the paper.

* Corresponding author. University College London, London, UK. Tel. +44 7595189982

Fax: +44 2089055855

E-mail address: arjun.nathan.11@ucl.ac.uk (A. Nathan).
\end{abstract}


were $36.7 \%$ and $29.1 \%(p=0.687)$ at follow-up, pad-free continence rates were $53.1 \%$ and $89.3 \%$ at $2 \operatorname{yr}(p<0.001)$, and ED rates were $98 \%$ and $93 \%(p=0.214)$, respectively. Conclusions: Salvage RARP has similar perioperative outcomes to primary RARP with inferior potency rates. Post-focal therapy sRARP has similar recurrence and continence rates to primary RARP. Post-whole gland therapy, complication, and recurrence rates are higher, and there is a higher risk of urinary incontinence. Patient summary: We report the largest propensity-matched comparison of salvage robot-assisted radical prostatectomy (RARP) after focal and whole gland therapy. Salvage RARP is a feasible procedure for the treatment of locally recurrent prostate cancer in high-volume centres; however, patients should be counselled appropriately as to the different outcomes.

(c) 2021 The Author(s). Published by Elsevier B.V. on behalf of European Association of

Urology. This is an open access article under the CC BY-NC-ND license (http:// creativecommons.org/licenses/by-nc-nd/4.0/).

\section{Introduction}

Nonsurgical primary treatment for prostate cancer (PCa) includes whole gland therapy such as radiotherapy or brachytherapy, and focal gland therapy such as highintensity focused ultrasound (HIFU), cryotherapy, or electroporation. Biochemical recurrence (BCR) and clinical recurrence rates for nonsurgical primary therapy can range from $20 \%$ to $70 \%$, and represent a significant risk of metastatic progression and cancer-specific mortality [1,2]. Salvage radical prostatectomy (sRP) is a potential treatment option for patients who have locally recurrent PCa.

Historically, sRP has shown inferior oncological and functional outcomes to primary radical prostatectomy with higher postoperative complication rates. The poor outcomes associated with SRP may be due to the technical hazards of surgery such as adhesions, fibrosis, poor tissue quality, and distortion of surgical planes that result from the primary treatment modality [3].

Outcomes for sRP have improved with the use of minimally invasive robot-assisted techniques. Salvage robot-assisted radical prostatectomy (sRARP) techniques have been developed to make the procedure more feasible with improved outcomes. Robotic procedures have the benefit of better vision and dexterity [4].

There is limited literature on sRARP as it is an uncommon and technically challenging procedure. Most studies are descriptive and therefore unable to assess whether outcomes of sRARP are comparable with those of RARP when adjusted for covariates such as age, body mass index (BMI), comorbidities, preoperative prostate-specific antigen (PSA), Gleason score, and clinical T stage. Despite a lack of level 1 evidence and the American and European urology associations describing focal therapy as experimental or investigational, the use of focal therapy for the treatment of local PCa is growing in popularity due to its perceived favourable side-effect profile compared with RARP [5-7]. However, the outcomes of salvage surgery after focal therapy have not been well compared with primary RARP. Clearly, it is not possible to randomise between primary RARP and sRARP, as these are different disease states, and therefore, the next best level of evidence can be obtained only by comparing risk-matched cohorts of sRARP with those of primary RARP.

Herein, we compared the perioperative, oncological, and functional outcomes of patients undergoing sRARP with those of propensity-matched patients undergoing primary RARP. Furthermore, we compared sRARP outcomes in patients who had previous primary whole gland therapy with those of patients having focal gland therapy.

\section{Patients and methods}

\subsection{Study population}

A total of 3852 consecutive patients underwent primary RARP or SRARP in a single, high-volume tertiary centre between January 2012 and March 2020, and had a minimum follow-up of 6 mo. Of these patients, 135 underwent sRARP after whole gland therapies such as radiotherapy, brachytherapy, and whole gland HIFU, and focal gland therapies such as focal HIFU, cryotherapy, and electroporation.

Primary treatment failure was defined as nadir plus $2 \mathrm{ng} / \mathrm{ml}$ following radiotherapy and three serial rises in PSA following minimally invasive treatments [6]. All patients had biopsy-proven local disease as well as staging investigations including multiparametric magnetic resonance imaging, bone scan, computed tomography, and prostatespecific membrane antigen positron emission tomography scans. Patients who had primary radiotherapy routinely received pelvic lymph node radiation, and therefore no pelvic lymph node dissection (PLND) was undertaken in this cohort in an attempt to prevent vascular and lymphatic complications [8]. In all other patients, PLND was decided based on the Briganti nomogram and European Association of Urology (EAU) guidelines, and in conjunction with informed patient choice [6].

\subsection{Data collection}

Data were collected retrospectively using local cancer registry databases, electronic medical records, general practitioner records, and patient questionnaires. Preoperative data including age, BMI, American Society of Anaesthesiologists (ASA) score, non-age-adjusted Charlson comorbidity index (CCI), PSA, T stage, Gleason score, EAU risk classification, and primary therapy were collected [9]. PSA, T stage, and Gleason score were collected after the primary treatment and before the surgical intervention. Perioperative data including operative time, estimated blood loss (EBL), blood transfusion, nerve sparing, PLND, length of stay (LOS), and 30-d Clavien-Dindo complications were also collected, as were postoperative oncological data including positive surgical margins 
(PSMs), T stage, Gleason score, BCR, clinical recurrence, and the use of further androgen deprivation therapy (ADT), radiotherapy, and chemotherapy. PSMs $<3$ or $>3 \mathrm{~mm}$ were reported as negative and positive [10]. BCR was defined as PSA $>0.2 \mathrm{ng} / \mathrm{ml}$ [6]. Functional data including full continence, defined as zero pads per day, and social continence, defined as one pad per day, were collected at 3-, 6-, 12-, and 24-mo intervals. Data on ED, defined as inability to have sexual function on at least $50 \%$ of attempts without the use of invasive aids, and survival at the last follow-up were also collected.

\subsection{Statistical analysis}

Data analysis was performed in IBM SPSS Statistics Data Editor version 23 (IBM Corporation, Armonk, NY, USA). To control for potential confounders, propensity scoring was used to achieve 135 1:1 matched samples from 135 sRARP cases [11]. Cases were matched by estimated propensity score to their nearest neighbour within a caliper distance of 0.02. Propensity scores were calculated using a logistic regression model considering the following baseline covariables known to influence outcomes: age, BMI, CCI, PSA, and T stage. Gleason score was avoided due to the unreliability in the tissue after prior treatment. All significance tests performed were two tailed. The independent $t$ test, Mann-Whitney $U$ test, and chi square test were used to evaluate differences between the primary and salvage groups. Kaplan-Meier survival analysis and log-rank tests were used to determine time to recurrence.

\section{Results}

\subsection{Primary versus salvage}

\subsubsection{Characteristics}

Full clinical and pathological characteristics are shown in Table 1. There were no statistical differences between age, BMI, ASA score, CCI, preoperative PSA, T stage, and Gleason score between the primary and salvage groups. Of the patients, $0 \%, 24.4 \%, 75.6 \%$ in the salvage group and $0 \%, 19.3 \%$, and $80.7 \%$ in the primary group were classified as having low, intermediate, and high risk, respectively, according to EAU risk stratification ( $p=0.307$; Table 1$)$. Two patients received whole gland radiotherapy followed by focal gland HIFU prior to sRARP. Eight patients had two focal treatments before undergoing sRARP: four had two HIFU treatments, two had HIFU followed by cryotherapy, and two had electroporation followed by HIFU.

\subsubsection{Perioperative}

The median operation time was $165 \mathrm{~min}$ in the salvage group and $140 \mathrm{~min}$ in the primary group $(p=0.004)$. Unilateral and bilateral nerve sparing were feasible, respectively, in $23 \%$ and $3.7 \%$ in the salvage group compared with $28.1 \%$ and $20.7 \%$ in the primary group $(p<0.001)$ due to technical feasibility and tumour location. There were fewer PLND procedures in the salvage group as zero patients undergoing primary radiotherapy received PLND due to safety concerns. There were no statistical differences in EBL, blood transfusion, or LOS between the primary and salvage groups. There was no statistically significant difference in the 30-d Clavien-Dindo III-V complication rates between salvage ( $1.5 \%$ ) and primary ( $0 \%$ ) surgery. Two patients in the salvage group suffered from grade $\geq$ III Clavien-Dindo complications requiring surgical intervention (Table 2).
One patient suffered from rectal injury with intraoperative primary repair following whole gland radiotherapy, and one patient suffered from a haematoma requiring reoperation and washout after HIFU therapy.

\subsubsection{Oncological}

The median follow-up in the salvage group was $521 \mathrm{~d}$ compared with $638 \mathrm{~d}$ in the primary group $(p=0.245)$. PSMs $<3$ and $>3 \mathrm{~mm}$ were $22.2 \%$ and $15.6 \%$, respectively, in the salvage group compared with $14.8 \%$ and $8.9 \%$, respectively, in the primary group $(p=0.022)$. Overall recurrence rates were $31.9 \%$ (biochemical $22.2 \%$, lesion $9.6 \%$ ) in the salvage group and $14.1 \%$ (biochemical $9.6 \%$, lesion $4.4 \%$ ) in the primary group $(p<0.001)$ at median follow-up. Recurrencefree survival rates are shown in Figure 1, with worse rates for the salvage cohort $(p<0.01)$. All patients with BCR or lesion recurrence had further ADT; $9.6 \%$ of the salvage patients who recurred had further salvage radiotherapy and $1.5 \%$ had chemotherapy (Table 3 ). PLND was undertaken in $18.5 \%$ of the patients in the salvage group and $43 \%$ in the primary group $(p<0.001)$. Overall survival was $95.6 \%$ in the salvage group compared with $99.3 \%$ in the primary group $(p=0.125)$.

\subsubsection{Functional}

Full continence was achieved at 3, 6, 12, and $24 \mathrm{mo}$, respectively, in $25.9 \%, 53.3 \%, 67.2 \%$, and $78.8 \%$ in the salvage group, compared with $25.9 \%, 43.7 \%, 71.6 \%$, and $84.3 \%$ in the primary group. There were no statistically significant differences between the groups. ED at the last follow-up was $94.8 \%$ in the salvage group compared with $76.3 \%$ in the primary group $(p<0.001$; Table 4$)$.

\subsection{Whole versus focal gland}

\subsubsection{Characteristics}

There were no statistically significant differences in age, BMI, ASA score, or CCI between the whole and focal gland salvage groups. There were more high-risk patients in the whole gland salvage group (83.7\%) than in the focal gland salvage group (70.9\%, $p=0.099$; Table 1). Full data comparing whole and focal gland treatment subtypes are available in the Supplementary Tables 1 and 2 .

\subsubsection{Perioperative}

Between whole and focal gland therapy, there were no statistically significant differences in operative time EBL, transfusion, or length of stay. Unilateral and bilateral nerve sparing were feasible, respectively, in $8.2 \%$ and $2 \%$ of the whole gland group compared with $31.4 \%$ and $4.7 \%$ of the focal group due to technical feasibility and tumour location $(p=0.001)$. Complication rates were significantly greater in the whole gland group (22\%) than in the focal group ( $8 \%$, $p=0.025$; Table 2).

\subsubsection{Oncological}

The median follow-up time in the whole gland group was $490 \mathrm{~d}$ compared with $542 \mathrm{~d}$ in the focal group $(p=0.669)$. PSMs $<3$ and $>3 \mathrm{~mm}$ were, respectively, $16.3 \%$ and $18.4 \%$ in 
Table 1 - Patient characteristics

\begin{tabular}{|c|c|c|c|c|c|c|c|c|c|c|}
\hline & \multicolumn{7}{|c|}{ Salvage subtypes } & \multirow[t]{2}{*}{ Salvage total } & \multirow[t]{2}{*}{ Primary total } & \multirow[t]{2}{*}{$p$ value } \\
\hline & Whole gland & Matched primary & $p$ value & Focal gland & Matched primary & $p$ value & Whole/focal $p$ value & & & \\
\hline Patients, $n$ & 49 & 49 & - & 86 & 86 & - & - & 135 & 135 & - \\
\hline Age (yr), median (IQR) & $70(63,73)$ & $68(65,74)$ & 0.765 & $70(64,72)$ & $69.5(67,73)$ & 0.118 & 0.509 & $70(64,73)$ & $69(66,73)$ & 0.158 \\
\hline BMI, median (IQR) & $27.7(26,30)$ & $28(27,30)$ & 0.285 & $27.8(26,30)$ & $28(26,29)$ & 0.751 & 0.798 & $27.7(25.9,30.6)$ & $28(26,29)$ & 0.736 \\
\hline \multicolumn{11}{|l|}{ ASA, $n(\%)$} \\
\hline 1 & $1(2.0)$ & 0 & 0.257 & $3(3.5)$ & $4(4.7)$ & 0.134 & 0.568 & $4(3.0)$ & $4(3.0)$ & 0.061 \\
\hline 2 & $43(87.8)$ & $48(98.0)$ & & $76(88.4)$ & $80(93.0)$ & & & $119(88.1)$ & $128(94.8)$ & \\
\hline 3 & $5(10.2)$ & $1(2.0)$ & & $7(8.1)$ & $2(2.3)$ & & & $12(8.9)$ & $3(2.2)$ & \\
\hline $\mathrm{CCI} \geq 1, n(\%)$ & $8(16.3)$ & $10(20.4)$ & 0.804 & $17(19.8)$ & $17(19.8)$ & $>0.999$ & $>0.999$ & $25(18.5)$ & $27(20.0)$ & $>0.999$ \\
\hline PSA preop, median (IQR) & $5.50(3.65,11.00)$ & $6.00(4.40,7.85)$ & 0.754 & $5.90(3.58,10.35)$ & $6.00(5.00,8.38)$ & 0.636 & 0.742 & $5.8(3.6,10.7)$ & $6(4.9,8.1)$ & 0.813 \\
\hline \multicolumn{11}{|l|}{ T stage preop, $n$ (\%) } \\
\hline $\mathrm{T} 1$ & $1(2.0)$ & $3(6.1)$ & 0.746 & $2(2.3)$ & $2(2.3)$ & 0.346 & 0.089 & $3(2.2)$ & $5(3.7)$ & 0.614 \\
\hline $\mathrm{T} 2$ & $21(42.9)$ & $19(38.8)$ & & $50(58.1)$ & $43(50.0)$ & & & $71(52.6)$ & $62(45.9)$ & \\
\hline $\mathrm{T} 3$ & $27(55.1)$ & $27(55.1)$ & & $34(39.5)$ & $41(47.7)$ & & & $61(45.2)$ & $68(50.4)$ & \\
\hline \multicolumn{11}{|l|}{ Gleason preop, $n$ (\%) } \\
\hline $3+3$ & $2(4.1)$ & 0 & 0.083 & $4(4.7)$ & 0 & 0.063 & 0.002 & $6(4.4)$ & $0(0)$ & 0.613 \\
\hline $3+4$ & $12(24.5)$ & $20(40.8)$ & & $42(48.8)$ & $37(43.0)$ & & & $54(40.0)$ & $57(42.2)$ & \\
\hline $3+5$ & 0 & $1(2.0)$ & & $1(1.2)$ & 0 & & & $1(0.7)$ & $1(0.7)$ & \\
\hline $4+3$ & $16(32.7)$ & $18(36.7)$ & & $23(26.7)$ & $24(27.9)$ & & & 39 (28.9) & $42(31.1)$ & \\
\hline $4+4$ & $8(16.3)$ & $4(8.2)$ & & $8(9.3)$ & 15 (17.4) & & & 16 (11.9) & 19 (14.1) & \\
\hline $4+5$ & $9(18.4)$ & $5(10.2)$ & & $8(9.3)$ & $8(9.3)$ & & & 17 (12.6) & $13(9.6)$ & \\
\hline $5+4$ & $2(4.1)$ & $1(2.0)$ & & 0 & $2(2.3)$ & & & $2(1.5)$ & $3(2.2)$ & \\
\hline EAU risk, $n(\%)$ & & & & & & & & & ) & \\
\hline Low & 0 & 0 & $>0.999$ & 0 & 0 & 0.223 & 0.099 & 0 & 0 & 0.307 \\
\hline Intermediate & $8(16.3)$ & $8(16.3)$ & & $25(29.1)$ & $18(20.9)$ & & & $33(24.4)$ & $26(19.3)$ & \\
\hline High & $41(83.7)$ & $41(83.7)$ & & $61(70.9)$ & $68(79.1)$ & & & $102(75.6)$ & $109(80.7)$ & \\
\hline
\end{tabular}




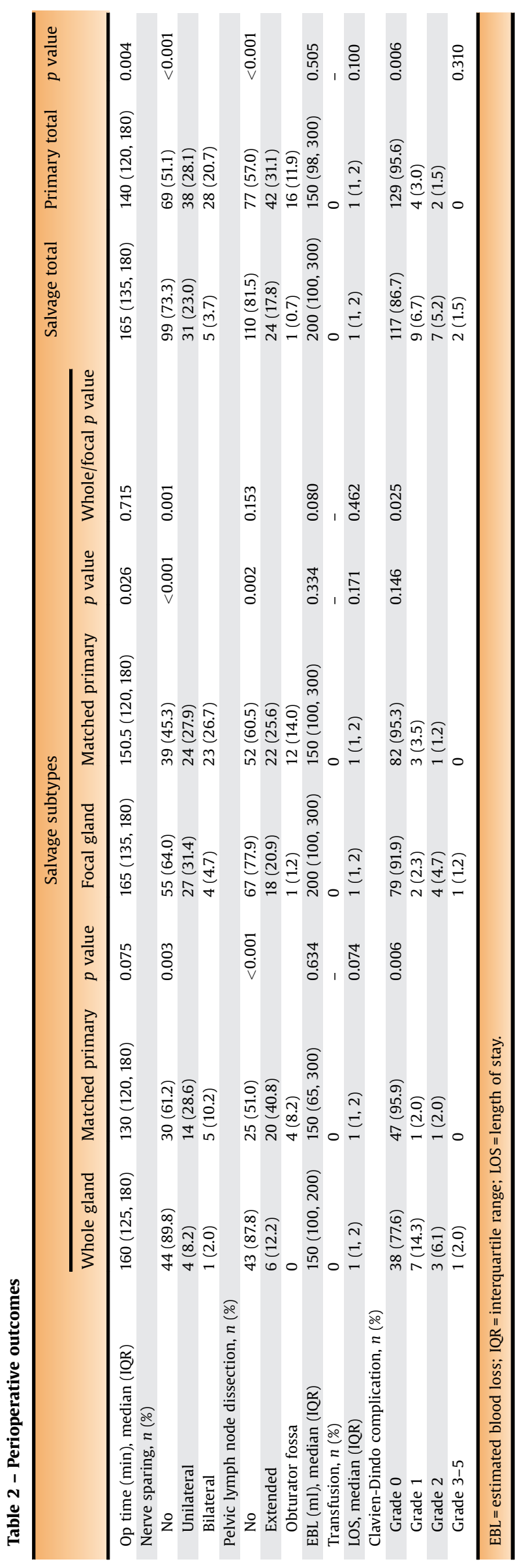

the whole group, compared with $25.6 \%$ and $14 \%$ in the focal group ( $p=0.787$ ). Recurrence occurred in $36.7 \%$ (biochemical $22.4 \%$ and lesion $14.3 \%$ ) of whole gland cases and $29.1 \%$ (biochemical $22.1 \%$ and lesion $7 \%$ ) of focal cases ( $p=0.687$ ) at median follow-up. Recurrence-free survival rates for whole and focal gland therapy are shown in Figure 2. Overall survival was $89.8 \%$ in the whole gland group compared with $98.8 \%$ in the focal group at the last follow-up ( $p=0.014$; Table 3).

\subsubsection{Functional}

In the whole gland group, full continence was achieved at 3 , 6,12 , and $24 \mathrm{mo}$ in $14.3 \%, 32.7 \%, 48.9 \%$, and $53.1 \%$, respectively, compared with $32.6 \%, 65.1 \%, 77.8 \%$, and $89.3 \%$ in the focal group. Full continence rates were statistically significantly greater in the focal group than in the whole gland group $(p=0.02,0.001,0.001$, and 0.001 , respectively) at 3, 6, 12, and $24 \mathrm{mo}$. ED at the last follow-up was $98 \%$ in the whole gland group compared with $93.0 \%$ in the focal gland group ( $p=0.214$; Table 4$)$.

\section{Discussion}

To our knowledge, this is the largest study globally comparing propensity-matched cohorts of sRARP with those of primary RARP. We discuss our findings in comparison with the findings of other descriptive studies and previous small-scale matched studies. We have further evaluated the outcomes of salvage surgery following primary whole gland treatments compared with focal treatments.

\subsection{Feasibility}

Our grade III-V Clavien-Dindo complication rate of $1.5 \%$ in the sRARP cohort is lower than that reported in previous literature (6-33\%) [12]. Our complication rates were statistically greater after whole gland therapy $(22 \%)$ than after focal therapy $(8 \%, p=0.025)$. Furthermore, when comparing salvage surgery after whole gland therapy with the matched primary cohort, the complication rate was significantly higher ( $22 \%$ vs $4 \%, p=0.006)$; however, this was not the case for salvage surgery after focal gland therapy ( $8 \%$ vs $5 \%, p=0.146$ ). Previous literature has shown that the complication rates can be up to $33 \%$ after whole gland and $9 \%$ after focal therapy $[13,14]$. Complication rates after sRARP are not statistically greater than primary RARP; however, they are clinically relevant. The retrospective nature of data collection may underestimate the complication rate in both groups. Our superior perioperative outcomes in comparison with literature may be due to the use of robot-assisted surgery and our experienced surgeons operating in a high-volume centre.

\subsection{Oncological outcomes}

PSM rates in the salvage group were significantly higher than those in the primary group. However, there was no difference between the whole and focal gland groups. Our 


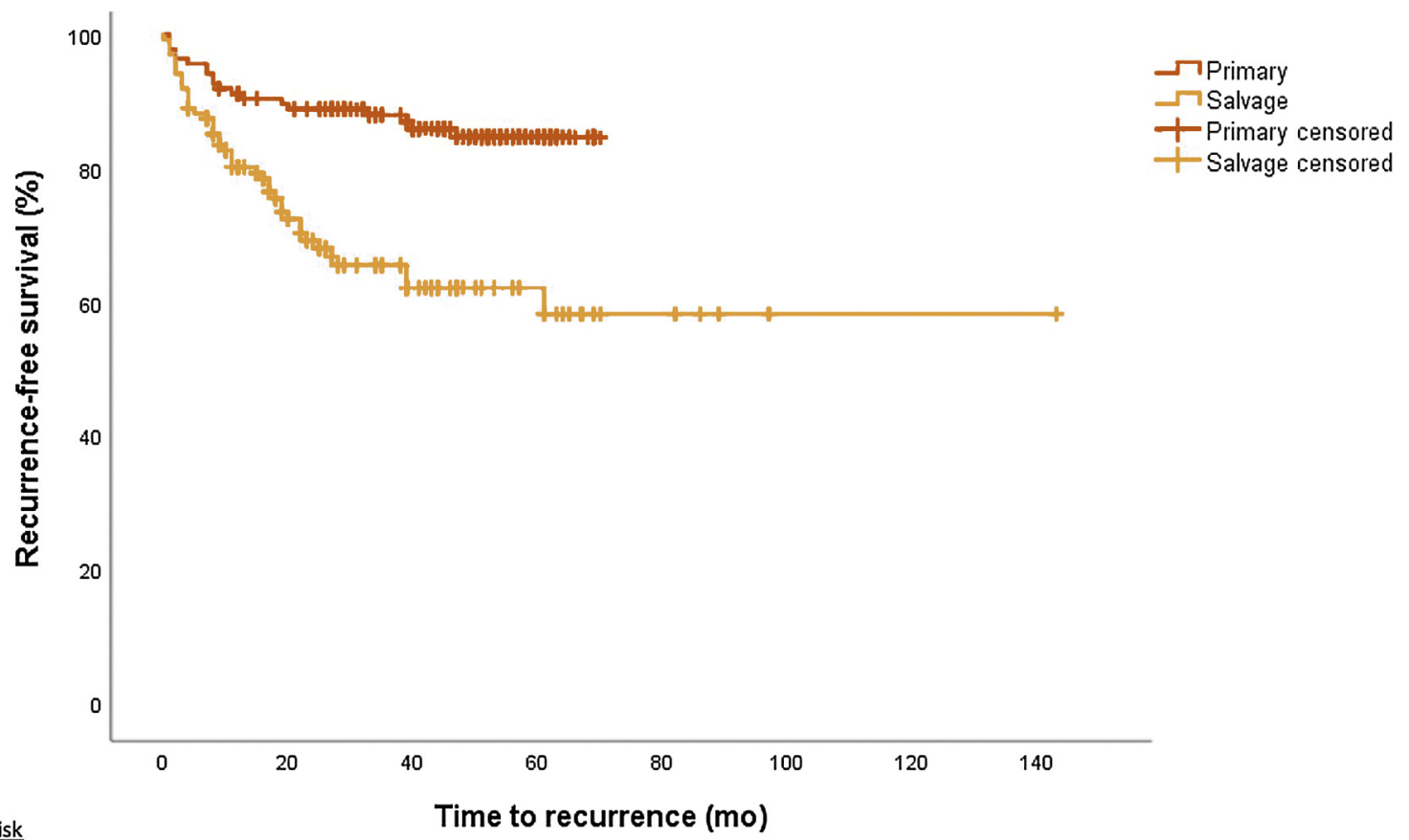

Number at risk

recurrence (mo)

$\begin{array}{lrrrr}\text { Primary } & 135 & 116 & 85 & 26 \\ \text { Salvage } & 135 & 71 & 34 & 16\end{array}$

5

1

1

1

Fig. 1 - Recurrence-free survival for primary RARP versus $\operatorname{SRARP}(p<0.001)$.

RARP = robot-assisted radical prostatectomy; $S R A R P=$ salvage RARP.

recurrence rates were significantly greater in the salvage group (31.9\%) than in the primary group (14.1\%) at 22 mo of follow-up ( $p<0.001)$. Furthermore, recurrence-free survival was inferior in the salvage group than in the primary group $(p<0.001)$. Furthermore, when comparing salvage surgery after whole gland therapy with the matched primary cohort, the recurrence rate was significantly higher (36.7\% vs $12.2 \%, p=0.004$ ); however, this was not the case for salvage surgery after focal gland therapy (29.1\% vs $15.1 \%$, $p=0.059$ ). All patients who suffered from recurrence had further ADT, and 9.6\% had further radiotherapy. Bates et al [15] propensity matched 53 sRARP patients to primary RARP and found a BCR rate of 33\% in the salvage group compared with $15 \%$ in the primary group at 26 mo ( $p$ $=0.029$ ). However, our sRARP cohort included 77\% EAU high-risk and 23\% intermediate-risk patients, compared with 26\% high-risk and 28\% intermediate-risk patients in Bates et al's [15] study. Our recurrence rates are similar to those in other studies with medium-term follow-up. Kaffenberger et al [16] report $18 \%$ BCR at 16 mo and Nunes-Silva et al [14] report $46 \%$ at $24 \mathrm{mo}$. In the largest systematic review of sRP, Chade et al [12] reported 5- and 10-yr BCR-free survival of 47-82\% and 28-53\%, respectively.

On comparison of patients who had recurrence with recurrence-free patients, we found preoperative PSA (7.2 vs $5.5 \mathrm{ng} / \mathrm{ml}$ ) and T stage (T2 29\%, T3 71\% vs T2 47\%, T3 53\%) to be higher. EAU guidelines suggest that referral for salvage therapy should be made when the PSA value is below 10 [6]. We would recommend that patients be referred for salvage treatment at an earlier stage immediately following biochemical failure. Further studies are required to identify the ideal PSA and T-stage threshold to deliver better sRARP oncological outcomes.

\subsection{Functional outcomes}

Owing to tumour location, loss of tissue planes, tissue adherence, as well as pre-existing ED, nerve sparing was feasible only in $27 \%$ of the salvage cohort compared with $49 \%$ in the primary cohort $(p<0.001)$. We found that continence improves with time in the salvage cohort, and patients should be counselled carefully about this. At 3, 6, 12 , and $24 \mathrm{mo}$, full continence was achieved in $26 \%, 53 \%$, $67 \%$, and $79 \%$, respectively. Continence rates are also superior for salvage surgery after focal therapy to those for salvage surgery after whole gland therapy. With better surgical reconstruction techniques and Retzius-sparing technique, continence rates have improved over time for RARP [17-19]. In 2004, Stephenson et al [20] reported a 5-yr sRARP continence rate of $39 \%$ after radiotherapy. However, more recent work in 2010 showed continence rates of $71 \%$ for sRARP [21].

Salvage RARP after focal therapy had better continence outcomes than whole gland therapy with medium-term continence rates varying from $73 \%$ to $91 \%$ [14,22]. In our study, full continence rates were greater at all time intervals after focal therapy than after whole gland therapy. At $24 \mathrm{mo}$, full continence was $89 \%$ after focal 
Table 3 - Oncological outcomes

\begin{tabular}{|c|c|c|c|c|c|c|c|c|c|c|}
\hline & \multicolumn{7}{|c|}{ Salvage subtypes } & \multirow[t]{2}{*}{ Salvage total } & \multirow[t]{2}{*}{ Primary total } & \multirow[t]{2}{*}{$p$ value } \\
\hline & Whole gland & Matched primary & $p$ value & Focal gland & Matched primary & $p$ value & Whole/focal $p$ value & & & \\
\hline Follow-up (d0, median (IQR) & $490(261,1053)$ & $530(328,841)$ & 0.702 & $541.5(312,890)$ & $649(401,1109)$ & 0.096 & 0.669 & $521(304,951)$ & $638(394,951)$ & 0.245 \\
\hline \multicolumn{11}{|l|}{ Surgical margins, $n(\%)$} \\
\hline Negative & $32(65.3)$ & $39(79.6)$ & 0.087 & $52(60.5)$ & $64(74.4)$ & 0.117 & 0.787 & $84(62.2)$ & $103(76.3)$ & 0.022 \\
\hline Positive $<3 \mathrm{~mm}$ & $8(16.3)$ & $7(14.3)$ & & $22(25.6)$ & $13(15.1)$ & & & $30(22.2)$ & $20(14.8)$ & \\
\hline Positive >3 mm & $9(18.4)$ & $3(6.1)$ & & $12(14.0)$ & $9(10.5)$ & & & $21(15.6)$ & $12(8.9)$ & \\
\hline \multicolumn{11}{|l|}{ T stage postop, $n(\%)$} \\
\hline $\mathrm{T} 1$ & 0 & 0 & 0.317 & 0 & 0 & 0.467 & 0.069 & 0 & 0 & 0.339 \\
\hline $\mathrm{T} 2$ & $16(32.7)$ & $21(42.9)$ & & $42(48.8)$ & $45(52.3)$ & & & $58(43.0)$ & $66(48.9)$ & \\
\hline T3 & $33(67.3)$ & $28(57.1)$ & & $44(51.2)$ & $41(47.7)$ & & & $77(57.0)$ & $69(51.1)$ & \\
\hline Upstaging, $n(\%)$ & $20(40.8)$ & $17(34.7)$ & 0.678 & $40(46.5)$ & $31(36.0)$ & 0.253 & 0.522 & $60(44.4)$ & 48 (35.6) & 0.195 \\
\hline \multicolumn{11}{|l|}{ Gleason postop, $n$ (\%) } \\
\hline $3+3$ & 0 & $1(2.0)$ & 0.467 & $1(1.2)$ & $3(3.5)$ & 0.427 & 0.016 & $1(0.7)$ & $4(3.0)$ & 0.288 \\
\hline $3+4$ & $17(34.7)$ & $17(34.7)$ & & $46(53.5)$ & $44(51.2)$ & & & $63(46.7)$ & $61(45.2)$ & \\
\hline $3+5$ & 0 & 0 & & 0 & 0 & & & 0 & 0 & \\
\hline $4+3$ & $19(38.8)$ & $14(28.6)$ & & $26(30.2)$ & $18(20.9)$ & & & $45(33.3)$ & $32(23.7)$ & \\
\hline $4+4$ & $3(6.1)$ & $1(2.0)$ & & $5(5.8)$ & $7(8.1)$ & & & $8(5.9)$ & $8(5.9)$ & \\
\hline $4+5$ & $10(20.4)$ & $13(26.5)$ & & $8(9.3)$ & $12(14.0)$ & & & $18(13.3)$ & $25(18.5)$ & \\
\hline $5+4$ & 0 & $3(6.1)$ & & 0 & $2(2.3)$ & & & 0 & $5(3.7)$ & \\
\hline Upgrading, $n(\%)$ & 15 (30.6) & $13(26.5)$ & 0.839 & $14(16.3)$ & $13(15.1)$ & $>0.999$ & 0.051 & $29(21.5)$ & $26(19.3)$ & 0.779 \\
\hline Total recurrence, $n$ (\%) & $18(36.7)$ & $6(12.2)$ & 0.004 & $25(29.1)$ & $13(15.1)$ & 0.059 & 0.687 & 43 (31.9) & $19(14.1)$ & 0.001 \\
\hline Biochemical only & $11(22.4)$ & $4(8.2)$ & & $19(22.1)$ & $9(10.5)$ & & & $30(22.2)$ & $13(9.6)$ & \\
\hline Lesion & $7(14.3)$ & $2(4.1)$ & & $6(7.0)$ & $4(4.7)$ & & & $13(9.6)$ & $6(4.4)$ & \\
\hline Further ADT, $n(\%)$ & $18(36.7)$ & $7(14.3)$ & 0.013 & $26(30.2)$ & $14(16.3)$ & 0.059 & 0.438 & $44(32.6)$ & $21(15.6)$ & 0.002 \\
\hline Further radiotherapy, $n$ (\%) & $2(4.1)$ & 0 & 0.500 & $11(12.8)$ & 0 & 0.001 & 0.099 & $13(9.6)$ & 0 & $<0.001$ \\
\hline Further chemotherapy, $n$ (\%) & $1(1.7)$ & 0 & $>0.999$ & $1(1.2)$ & 0 & $>0.999$ & 0.685 & $2(1.5)$ & 0 & 0.500 \\
\hline Overall survival, $n(\%)$ & $44(89.8)$ & $49(100)$ & 0.063 & $85(98.8)$ & $85(98.8)$ & $>0.999$ & 0.014 & $129(95.6)$ & $134(99.3)$ & 0.125 \\
\hline
\end{tabular}


Table 4 - Functional outcomes

\begin{tabular}{|c|c|c|c|c|c|c|c|c|c|c|}
\hline & \multicolumn{7}{|c|}{ Salvage subtypes } & \multirow{2}{*}{$\begin{array}{c}\text { Salvage } \\
\text { total }\end{array}$} & \multirow{2}{*}{$\begin{array}{c}\text { Primary } \\
\text { total }\end{array}$} & \multirow[t]{2}{*}{$p$ value } \\
\hline & $\begin{array}{l}\text { Whole } \\
\text { gland }\end{array}$ & $\begin{array}{l}\text { Matched } \\
\text { primary }\end{array}$ & $p$ value & $\begin{array}{l}\text { Focal } \\
\text { gland }\end{array}$ & $\begin{array}{l}\text { Matched } \\
\text { primary }\end{array}$ & $p$ value & $\begin{array}{c}\text { Whole/focal } \\
\quad p \text { value }\end{array}$ & & & \\
\hline \multicolumn{11}{|l|}{ Full continence, $n(\%)$} \\
\hline $3 \mathrm{mo}$ & $7(14.3)$ & $10(20.4)$ & 0.607 & $28(32.6)$ & $25(29.1)$ & 0.742 & 0.020 & $35(25.9)$ & 35 (25.9) & $>0.999$ \\
\hline $6 \mathrm{mo}$ & $16(32.7)$ & $21(42.9)$ & 0.424 & $56(65.1)$ & $38(44.2)$ & 0.007 & $<0.001$ & $72(53.3)$ & $59(43.7)$ & 0.137 \\
\hline $12 \mathrm{mo}$ & $23(48.9)$ & 35 (72.9) & 0.043 & $63(77.8)$ & $61(70.9)$ & 0.345 & 0.001 & $86(67.2)$ & $96(71.6)$ & 0.583 \\
\hline $24 \mathrm{mo}$ & $26(53.1)$ & $41(85.4)$ & 0.027 & $67(89.3)$ & $72(83.7)$ & 0.481 & $<0.001$ & $93(78.8)$ & $113(84.3)$ & 0.337 \\
\hline \multicolumn{11}{|l|}{ Social continence, $n$ (\%) } \\
\hline $3 \mathrm{mo}$ & $10(20.4)$ & $30(61.2)$ & $<0.001$ & $46(53.5)$ & $49(57.0)$ & 0.749 & $<0.001$ & $56(41.5)$ & $79(58.5)$ & 0.007 \\
\hline $6 \mathrm{mo}$ & $27(55.1)$ & $37(75.5)$ & 0.078 & $71(82.6)$ & $62(72.1)$ & 0.164 & 0.001 & $98(71.0)$ & $99(73.3)$ & $>0.999$ \\
\hline $12 \mathrm{mo}$ & $30(61.2)$ & $43(87.8)$ & 0.011 & 79 (91.9) & $74(86.0)$ & 0.332 & $<0.001$ & $109(80.7)$ & $117(86.7)$ & 0.268 \\
\hline $24 \mathrm{mo}$ & 39 (79.6) & 45 (91.8) & 0.180 & $81(94.2)$ & $83(96.5)$ & 0.687 & 0.010 & $120(88.9)$ & $128(94.8)$ & 0.115 \\
\hline Erectile dysfunction, $n(\%)$ & $48(98.0)$ & $36(73.5)$ & 0.002 & $80(93.0)$ & $67(77.9)$ & 0.015 & 0.214 & $128(94.8)$ & $103(76.3)$ & $<0.001$ \\
\hline
\end{tabular}

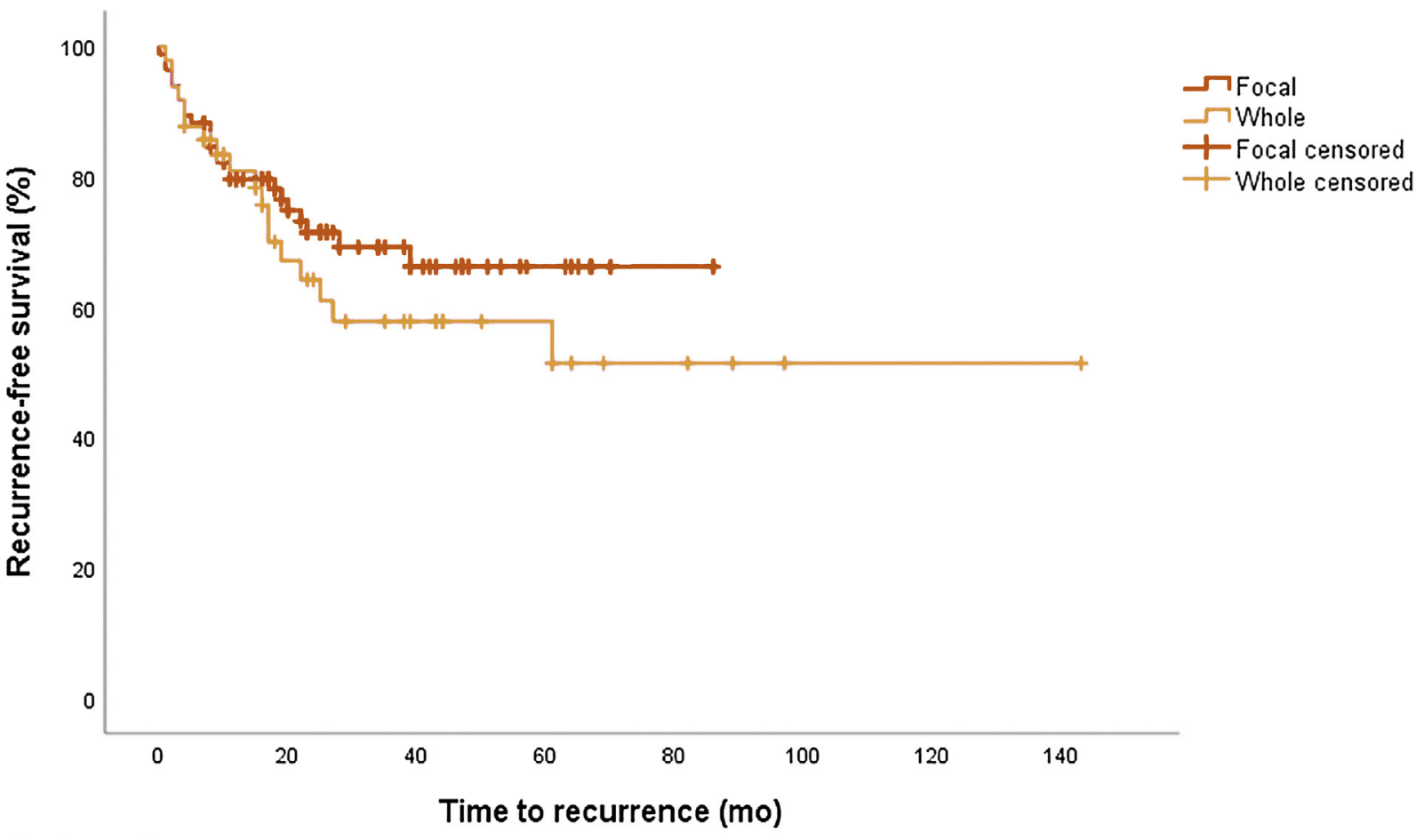

Number at risk

$\begin{array}{llllll}\text { Focal } & 86 & 47 & 20 & 7 & 1 \\ \text { Whole } & 49 & & & & \end{array}$

Fig. 2 - Recurrence-free survival of sRARP whole gland versus focal gland treatment $(p=0.375)$. SRARP = salvage robot-assisted radical prostatectomy.

gland therapy and 53\% after whole gland therapy ( $p$ $<0.001$ ). Onol et al [23] found poor ED rates similar to those of our study, but improved continence outcomes after focal therapy compared with those after whole gland therapy. Our data show a significantly greater rate of ED of $95 \%$ in the salvage group compared with $77 \%$ in the primary group $(p<0.001)$. ED rates up to $100 \%$ are widely reported after sRARP due to the resection of the neurovascular architecture $[12,15,16]$.

\subsection{Limitations}

Our study is the largest propensity-matched comparison of sRARP; however, this is a retrospective, single-centre study 
with medium-term follow-up. Patients who had previous primary treatment and then RARP will have different preoperative pathology from patients who receive primary RARP; however propensity score matching has been attempted to control these variations. Further, all surgeries were undertaken in a high-volume centre by experienced surgeons. Larger, multicentre prospective studies with longer-term follow-up are needed to better appreciate the outcomes of sRARP.

\section{Conclusions}

Salvage RARP is a feasible operation with perioperative outcomes comparable with those of primary RARP in similar propensity-matched cohorts. Recurrence rates are higher with sRARP, and this may be due to the high-risk, aggressive characteristics of our sRARP cohort. Continence rates are comparable between the primary and salvage cohorts, although ED rates are inferior in the salvage group. Salvage RARP after whole gland therapy shows inferior continence rates to focal therapy and a matched primary RARP cohort. Perioperative outcomes are acceptable; however, complication rates are higher after whole gland therapy. In high-volume centres, SRARP is a safe and feasible treatment for locally recurrent PCa, but patients should be counselled about poorer outcomes compared with primary RARP, especially with regard to erectile function and particularly in those who received primary whole gland therapy.

\footnotetext{
Author contributions: Arjun Nathan had full access to all the data in the study and takes responsibility for the integrity of the data and the accuracy of the data analysis.
}

Study concept and design: A. Nathan, De Groote, S. Nathan. Acquisition of data: A. Nathan, Arora, Phuah, Flora, Patel. Analysis and interpretation of data: A. Nathan, Fricker. Drafting of the manuscript: A. Nathan.

Critical revision of the manuscript for important intellectual content: Kasivisvanathan, Sridhar, Shaw, Kelly, Briggs, Rajan, Sooriakumaran, S. Nathan.

Statistical analysis: A. Nathan, Fricker.

Obtaining funding: None.

Administrative, technical, or material support: None.

Supervision: Sridhar, Shaw, Kelly, Briggs, Rajan, Sooriakumaran, S. Nathan.

Other: None.

Financial disclosures: Arjun Nathan certifies that all conflicts of interest, including specific financial interests and relationships and affiliations relevant to the subject matter or materials discussed in the manuscript (eg, employment/affiliation, grants or funding, consultancies, honoraria, stock ownership or options, expert testimony, royalties, or patents filed, received, or pending), are the following: None.

Funding/Support and role of the sponsor: The first-author, Arjun Nathan, is supported by the National Institute for Health Research.

Ethics statement: All patients were registered as part of the British Association of Urological Surgeons (BAUS) national outcomes audit and registered with the institutional audit departments of University College London Hospitals NHS Foundation Trust. Ethical guidance was followed in line with the institutional clinical governance protocols. All procedures followed were in accordance with the ethical standards of the responsible committee on human experimentation (institutional and national) and with the Helsinki Declaration of 1975, as revised in 2000.

Acknowledgements: The authors acknowledge Uro-oncology Department, University College London Hospitals NHS Foundation Trust, London, UK, and Division of Surgery and Interventional Science, University College London, London, UK.

\section{CRediT authorship contribution statement}

Arjun Nathan: Conceptualization, Methodology, Investigation, Data curation, Writing - original draft. Monty Fricker: Formal analysis. Ruben De Groote: Conceptualization, Methodology, Writing - review \& editing. Amandeep Arora: Investigation. Yuzhi Phuah: Investigation. Kiran Flora: Investigation. Sonam Patel: Investigation. Veeru Kasivisvanathan: Writing - review \& editing. Ashwin Sridhar: Supervision, Writing - review \& editing. Greg Shaw: Supervision, Writing - review \& editing. John Kelly: Supervision, Writing - review \& editing. Tim Briggs: Supervision, Writing - review \& editing. Prabhakar Rajan: Supervision, Writing - review \& editing. Prasanna Sooriakumaran: Supervision, Writing - review \& editing. Senthil Nathan: Supervision, Writing - review \& editing.

\section{Appendix A. Supplementary data}

Supplementary material related to this article can be found, in the online version, at doi:https://doi.org/10.1016/j. euros.2021.03.003.

\section{References}

[1] Agarwal PK, Sadetsky N, Konety BR, Resnick MI, Carroll PR. Cancer of the Prostate Strategic Urological Research Endeavor (CaPSURE). Treatment failure after primary and salvage therapy for prostate cancer-likelihood, patterns of care, and outcomes. Cancer 2008;112:307-14.

[2] Chade DC, Shariat SF, Cronin AM, et al. Salvage radical prostatectomy for radiation-recurrent prostate cancer: a multi-institutional collaboration. Eur Urol 2011;60:205-10.

[3] Coelho RF, Patel MB, Chauhan S, et al. Salvage robotic-assisted radical prostatectomy (SRALP) for treatment of radio-recurrent prostate cancer: description of technique and multi-institutional outcomes. J Endourol 2010;24:A347.

[4] Kenney PA, Nawaf CB, Mustafa M, et al. Robotic-assisted laparoscopic versus open salvage radical prostatectomy following radiotherapy. Can J Urol 2016;23:8271-7.

[5] Ahmed HU, Hindley RG, Dickinson L, et al. Focal therapy for localised unifocal and multifocal prostate cancer: a prospective development study. Lancet Oncol 2012;13:622-32.

[6] Mottet N, Bellmunt J, Bolla M, et al. EAU-ESTRO-SIOG guidelines on prostate cancer. Part 1: screening, diagnosis, and local treatment with curative intent. Eur Urol 2017;71:618-29.

[7] Sanda MG, Cadeddu JA, Kirkby E, et al. Clinically localized prostate cancer: AUA/ASTRO/SUO guideline. Part I: risk stratification, shared decision making, and care options. J Urol 2018;199:683-90.

[8] Keegan KA, Cookson MS. Complications of pelvic lymph node dissection for prostate cancer. Curr Urol Rep 2011;12:203-8. 
[9] Cooperberg MR, Pasta DJ, Elkin EP, et al. The University of California, San Francisco Cancer of the Prostate Risk Assessment score: a straightforward and reliable preoperative predictor of disease recurrence after radical prostatectomy. J Urol 2005;173:1938-42.

[10] Sooriakumaran P, Ploumidis A, Nyberg T, et al. The impact of length and location of positive margins in predicting biochemical recurrence after robot-assisted radical prostatectomy with a minimum follow-up of 5 years. BJU Int 2015;115:106-13.

[11] Rosenbaum PR, Rubin DB. The central role of the propensity score in observational studies for causal effects. Biometrika 1983;70:41-55.

[12] Chade DC, Eastham J, Graefen M, et al. Cancer control and functional outcomes of salvage radical prostatectomy for radiation-recurrent prostate cancer: a systematic review of the literature. Eur Urol 2012;61:961-71.

[13] Kimura M, Mouraviev V, Tsivian M, Mayes JM, Satoh T, Polascik TJ Current salvage methods for recurrent prostate cancer after failure of primary radiotherapy. BJU Int 2010;105:191-201.

[14] Nunes-Silva I, Barret E, Srougi V, et al. Effect of prior focal therapy on perioperative, oncologic and functional outcomes of salvage robotic assisted radical prostatectomy. J Urol 2017;198:1069-76.

[15] Bates AS, Samavedi S, Kumar A, et al. Salvage robot assisted radical prostatectomy: a propensity matched study of perioperative, oncological and functional outcomes. Eur J Surg Oncol 2015;41:1540-6.

[16] Kaffenberger SD, Keegan KA, Bansal NK, et al. Salvage robotic assisted laparoscopic radical prostatectomy: a single institution, 5-year experience. J Urol 2013;189:507-13.
[17] Ficarra V, Novara G, Rosen RC, et al. Systematic review and metaanalysis of studies reporting urinary continence recovery after robot-assisted radical prostatectomy. Eur Urol 2012;62:405-17.

[18] Sridhar AN, Abozaid M, Rajan P, et al. Surgical techniques to optimize early urinary continence recovery post robot assisted radical prostatectomy for prostate cancer. Curr Urol Rep 2017;18:71.

[19] Umari P, Eden C, Cahill D, Rizzo M, Eden D, Sooriakumaran P. Retzius-sparing versus standard robot-assisted radical prostatectomy: a comparative prospective study of nearly 500 patients. J Urol 2021;205:780-90.

[20] Stephenson AJ, Scardino PT, Bianco Jr FJ, DiBlasio CJ, Fearn PA, Eastham JA. Morbidity and functional outcomes of salvage radical prostatectomy for locally recurrent prostate cancer after radiation therapy. J Urol 2004;172(6 Pt 1):2239-43.

[21] Chauhan S, Patel MB, Coelho R, et al. Preliminary analysis of the feasibility and safety of salvage robot-assisted radical prostatectomy after radiation failure: multi-institutional perioperative and short-term functional outcomes. J Endourol 2011;25:1013-9.

[22] Herrera-Caceres JO, Nason GJ, Salgado-Sanmamed N, et al. Salvage radical prostatectomy following focal therapy: functional and oncological outcomes. BJU Int 2020;125:525-30.

[23] Onol FF, Bhat S, Moschovas M, et al. Comparison of outcomes of salvage robot-assisted laparoscopic prostatectomy for post-primary radiation vs focal therapy. BJU Int 2020;125:103-11. 